\title{
Development
}

\section{Poor Concordance of Floxed Sequence Recombination in Single Neural Stem Cells: Implications for Cell Autonomous Studies}

\author{
Tyler Joseph Dause ${ }^{1}$ and Elizabeth Diana Kirby ${ }^{1,2,3}$
}

https://doi.org/10.1523/ENEURO.0470-19.2020

${ }^{1}$ Deptartment of Psychology, The Ohio State University, Columbus, OH 43210, ${ }^{2}$ Deptartment of Neuroscience, The Ohio State University, Columbus, $\mathrm{OH} 43210$, and ${ }^{3}$ The Chronic Brain Injury Program, The Ohio State University, Columbus, $\mathrm{OH} 43210$

\section{Visual Abstract}

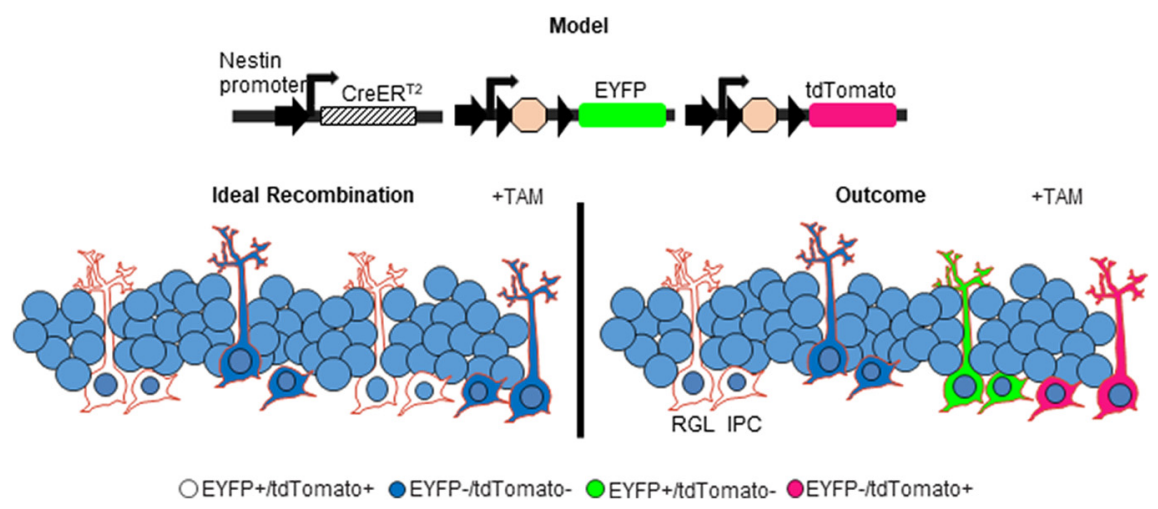

To manipulate target gene function in specific adult cell populations, tamoxifen (TAM)-dependent $\mathrm{CreER}^{\mathrm{T2}}$ is widely used to drive inducible, site-specific recombination of loxP flanked sequences. In studies of cell autonomous target gene function, it is common practice to combine these CreER ${ }^{\mathrm{T} 2}$-lox systems with a ubiquitously expressed stop-floxed fluorescent reporter gene to identify single cells supposedly undergoing target gene recombination. Here, we studied the reliability of using Cre-induced recombination of one gene to predict recombination in another gene at the single-cell level in adult hippocampal neural stem and progenitor cells (NSPCs). Using both probabilistic predictions in a generic experimental paradigm, as well as a mouse model with two separate stop-floxed reporters plus a Nestin promoter-driven $\mathrm{CreER}^{\mathrm{T} 2}$, we found that, in individual cells, recombination of one gene was a poor predictor of

\section{Significance Statement}

We investigate the reliability of a widely used transgenic mouse model in studies of adult neural stem and progenitor cells (NSPCs). Ligand-dependent Cre recombinases, such as the CreER ${ }^{\top 2}$ model, are a fundamental tool for inducible gene modification used to investigate gene function in many cell populations. It is common practice to combine NSPC-specific CreER ${ }^{T 2}$-lox systems with a ubiquitously expressed stopfloxed fluorescent reporter gene to identify single cells undergoing target gene recombination in studies of cell autonomous gene function. Our probabilistic predictions and experimental data suggest that use of stop-floxed reporters to investigate cell autonomous gene function in NSPCs may lead to false conclusions because recombination in separate genes can show poor concordance in individual cells. 
recombination in another. This poor concordance in floxed sequence recombination across genes suggests that use of stop-floxed reporters to investigate cell autonomous gene function may not be universally reliable and could lead to false conclusions.

Key words: adult neurogenesis; cell autonomous; CreER ${ }^{\mathrm{T} 2}$ recombinase; hippocampus; neural stem cells; subventricular zone

\section{Introduction}

In the adult mammalian brain, there are two primary neurogenic niches where neural stem and progenitor cells (NSPCs) proliferate throughout life: the subventricular zone (SVZ) and the dentate gyrus (DG) of the hippocampus (Gage, 2000). NSPCs in these regions create new neurons during adulthood, which incorporate into the preexisting circuitry in the olfactory bulb or DG, respectively, a process known as neurogenesis (Zhao et al., 2008). As evidence has mounted for the conservation of adult neurogenesis across species (Kempermann, 2015), understanding the functional properties of adult neurogenesis, as well as the molecular mechanisms that regulate it, has emerged as a major focus of research.

A common and powerful approach to dissecting molecular mediators of complex cellular processes like neurogenesis relies on gene ablation or overexpression to simulate gain or loss of function of target proteins. Genetic manipulation of adult neurogenesis requires cell-specific, inducible models that affect adult NSPCs selectively without influencing developmental counterparts. Ligand-dependent Cre recombinases, such as the tamoxifen (TAM)-dependent $\mathrm{CreER}^{\mathrm{T} 2}$, are widely-used for driving such inducible, site-specific recombination of loxP flanked sequences in adult NSPCs (Feil et al., 2009; Semerci and MaleticSavatic, 2016). Several CreER ${ }^{\mathrm{T} 2}$ mouse lines are currently available which use NSPC-specific promoters to drive Cre expression and therefore TAM-induced recombination in adult NSPCs (Sun et al., 2014; Yang et al., 2015; Semerci and Maletic-Savatic, 2016). In many studies, NSPC-targeted CreER ${ }^{\text {T2 }}$-lox systems are combined with a ubiquitously-expressed stop-floxed fluorescent reporter gene to confirm recombination in NSPCs (Sun et al., 2014; Kirby et al., 2015; Tannenholz et al., 2016). It is also common to use these fluorescent proteins more cell-specifically to investigate cell autonomous effects of recombination in the experimental gene of interest (Zimmermann et al., 2018; Zhou et al., 2018; Franz et al., 2019; Zhang et al., 2019).

Received November 13, 2019; accepted January 23, 2020; First published February 20, 2020.

The authors declare no competing financial interests.

Author contributions: T.J.D. and E.D.K. designed research; T.J.D. and E.D.K. performed research; T.J.D. and E.D.K. analyzed data; T.J.D. and E.D.K. wrote the paper.

This work was supported by the National Science Foundation Grant IOS1923094 (to E.D.K.)

Correspondence should be addressed to Elizabeth Diana Kirby at kirby. 224@osu.edu.

https://doi.org/10.1523/ENEURO.0470-19.2020

Copyright (C) 2020 Dause and Kirby

This is an open-access article distributed under the terms of the Creative Commons Attribution 4.0 International license, which permits unrestricted use, distribution and reproduction in any medium provided that the original work is properly attributed.
These cell autonomous experimental paradigms suppose that target gene and fluorescent reporter gene recombination occur in the same cell with high probability, allowing investigators to identify cell autonomous effects by comparing fluorescent and non-fluorescent cells.

To our knowledge, the assumption that fluorescent reporter expression reliably equates to recombination in a separate gene in the same cell has yet to be scrutinized. Within a cell, each recombination event is independent, and, with a transiently-activated Cre such as CreER ${ }^{\text {T2 }}$, divergence of recombination status across loci seems possible. Here, we examine the reliability of using Cre-induced recombination of one gene to predict recombination in another gene at the single-cell level in adult NSPCs. Using one common inducible Cre-driver mouse line and two, widelyused stop-floxed fluorescent reporter lines, we found that Cre-induced expression of one fluorescent reporter did not reliably predict expression of the other within a single cell in vivo. Given our results, we suggest that stop-floxed fluorescent reporters may misrepresent cell autonomous effects of gene recombination at a cell-specific level and that it may not be prudent to assume any specific pairing of reporter and target genes shows high recombination concordance in single cells without other, confirmatory data.

\section{Materials and Methods}

\section{Mice}

NestinCreER ${ }^{\mathrm{T} 2}$ mice (Jackson \#016261) were crossed with two conditional reporter lines: Rosa-stop-floxedEYFP (Srinivas et al., 2001; Jackson \#006148) and RosaCAG-stop-floxed-tdTomato (Madisen et al., 2010: Ai9; Jackson \#007909). Mice were bred and maintained in The Ohio State University Psychology building mouse vivarium in standard ventilated cages on a 12/12 h light/dark cycle (lights on 6:30 A.M.), with ad libitum access to food and water. Male and female mice were eight to nine weeks old at the time of the experiment and housed in groups of two to four. All animal use was in accordance with institutional guidelines approved by The Ohio State University Institutional Animal Care and Use Committee.

\section{TAM administration}

TAM was dissolved in sterile sunflower oil at $20 \mathrm{mg} / \mathrm{ml}$, overnight with agitation at $37^{\circ} \mathrm{C}$. TAM solution was stored at $+4^{\circ} \mathrm{C}$ for up to one week. TAM (or oil vehicle) was injected $(180 \mathrm{mg} / \mathrm{kg} / \mathrm{d}$, i.p.) for 3 or $5 \mathrm{~d}$.

\section{Immunofluorescent staining/antibodies}

After $2 \mathrm{~d}$ (3D short/5D mice) or $4 \mathrm{~d}$ (3D long mice), mice were anesthetized with an $87.5 \mathrm{mg} / \mathrm{kg}$ ketamine, $12.5 \mathrm{mg} /$ $\mathrm{kg}$ xylazine mixture and then transcardially perfused with ice-cold 0.1 M PBS. Harvested brains were fixed in 4\% 
Table 1: Primary and secondary antibodies

\begin{tabular}{|c|c|c|c|c|c|}
\hline Primary antibody & Vendor/ product no. & Dilution & Secondary & Vendor/product no. & Dilution \\
\hline Rabbit anti-mCherry & Abcam ab167453 & $1: 500$ & $\begin{array}{l}\text { Donkey anti-Rabbit lgG }(\mathrm{H}+\mathrm{L}) \\
\text { highly cross-adsorbed secondary } \\
\text { antibody, AlexaFluor } 555\end{array}$ & $\begin{array}{l}\text { ThermoFisher Scientific } \\
\text { A-31572 }\end{array}$ & $1: 500$ \\
\hline $\begin{array}{l}\text { Goat } \\
\text { Anti-GFP antibody }\end{array}$ & Abcam Ab6673 & $1: 1000$ & $\begin{array}{l}\text { Donkey anti-Goat lgG }(\mathrm{H}+\mathrm{L}) \text { cross- } \\
\text { adsorbed secondary antibody, } \\
\text { AlexaFluor } 488\end{array}$ & Fisher A-11055 & $1: 500$ \\
\hline $\begin{array}{l}\text { Rat } \\
\text { anti-Ki-67 monoclonal } \\
\text { antibody }\end{array}$ & $\begin{array}{l}\text { Invitrogen } \\
14-5698-82\end{array}$ & $1: 500$ & $\begin{array}{l}\text { AlexaFluor } 647 \text { AffiniPure donkey } \\
\text { anti-Rat IgG }(\mathrm{H}+\mathrm{L})(712-605-153)\end{array}$ & $\begin{array}{l}\text { Jackson } \\
\text { ImmunoResearch } \\
\text { 712-605-153 }\end{array}$ & $1: 500$ \\
\hline
\end{tabular}

paraformaldehyde in $0.1 \mathrm{M}$ phosphate buffer overnight at $4^{\circ} \mathrm{C}$. After equilibration in $30 \%$ sucrose in PBS, $40-\mu \mathrm{m}$ coronal brain sections were obtained in one in 12 series on a freezing microtome (Leica), and stored in cryoprotectant at $-20^{\circ} \mathrm{C}$ until use. Brain sections were rinsed with PBS three times then incubated in a blocking solution containing $1 \%$ normal donkey serum (Jackson ImmunoResearch) and $0.3 \%$ Triton X-100 (Acros) in PBS. Sections were then incubated in primary antibody (Table 1) diluted in blocking solution overnight at $4^{\circ} \mathrm{C}$ with rotation. The following day, after three rinses in PBS, cells were incubated in secondary antibodies (Table 1) diluted 1:500 in blocking solution for $2 \mathrm{~h}$ with rotation. The DG of the hippocampus was imaged in $15-\mu \mathrm{m}$ z-stacks at $20 \times$ magnification using a Zeiss Axio Observer Z.1 with apotome digital imaging system and Axiocam 506 monochrome camera (Zeiss).

\section{Automated image processing}

Colocalization of immunofluorescent signal was analyzed with just another colocalization plugin (JACoP) software on ImageJ (Bolte and Cordelières, 2006). First, z-stacks from each DG were separated into individual $1-\mu \mathrm{m}$-thick images, 1 image per fluorescent channel. Images were thresholded then overlapped using anatomic features. The JACoP plugin then re-stacked the image files and analyzed overlap of EYFP and tdTomato. These data were used to determine EYFP and tdTomato percent area, the proportion of EYFP overlapping tdTomato and the proportion of tdTomato overlapping EYFP.

\section{NSPC identification and manual cell counts}

Radial glia-like NSCs (RGLs) were identified by their $\mathrm{GFAP}+$ radial processes extending from the subgranular zone (SGZ) into the molecular layer, while cells with $\mathrm{Ki}$ $67+$ cell bodies in the SGZ layer were identified as intermediate progenitor cells (IPCs). All NSPCs were counted in each section. RGLs and IPCs were manually identified in 1- $\mu \mathrm{m}$ z-stack images using ImageJ then assessed for EYFP or tdTomato coexpression as described. Density of RGLs and IPCs was determined as the number of cells per area in the DG/SGZ. The SGZ was defined as the zone spanning two cell body widths between the dense granular cell layer and the hilus.

\section{Statistical analysis}

Comparisons of more than two groups were performed using one-way or two-way ANOVAs followed by Tukey's multiple comparisons. Correlations were performed using Pearson's correlation. One-sample $t$ tests were used to compare difference from a theoretical value of $100 \%$. All analyses were performed using Prism (v8.0; GraphPad Software), and $p<0.05$ was considered significant (Table 2; Extended Data Tables 2-1, 2-2).

\section{Results}

First, we explored the theoretical accuracy of using Cre-induced recombination of one gene to predict recombination in another gene. We chose to model an experimental paradigm where recombination of a stop-floxed fluorescent reporter (Gene R) is used as a marker of recombination in a target gene (Gene T) to make conclusions about the cell autonomous effect of target gene recombination (Fig. 1A). This design assumes that detection of reporter protein indicates target gene recombination with high probability (a true positive signal) and absence of reporter protein indicates lack of target gene recombination with high probability (a true negative signal; Fig. 1B).

To explore the consequences of deviations from the ideal of high true signal fraction, we performed probabilistic calculations of true signals based on several simplifying assumptions. First, we assumed recombination of gene $\mathrm{T}$ to be an all-or-none outcome (i.e., no possibility of heterozygosity). Next, we assumed that recombination does not occur in either gene when Cre is not expressed (i.e., minimal ectopic recombination). In many transgenic models, this assumption has been shown to hold true (Imayoshi et al., 2006; Kim et al., 2011; Yang et al., 2015). Third, we assumed that the probability of reporter recombination is larger than or approximately equal to the probability of target gene recombination $\left[P\left(R_{r}\right) \geq P\left(T_{r}\right)\right]$. This assumption is based on the likely selection of stop-floxed fluorescent reporter constructs that recombine with high efficiency. Given these constraints, $\mathrm{P}$ (total true) $=\mathrm{P}($ true +$)+\mathrm{P}($ true -$)=1+2 * \mathrm{P}\left(\mathrm{R}_{\mathrm{r}}\right) *$ $P\left(T_{r} \mid R_{r}\right)-P\left(R_{r}\right)-P\left(T_{r}\right)$ (equation derivation in Extended Data Fig. 1-1A).

In most experimental paradigms, $P\left(R_{r}\right)$ is known based on immunohistochemical quantification of percent of target cells expressing the reporter. For our estimates, we 
Table 2: Statistics table

\begin{tabular}{|c|c|c|c|c|c|c|c|}
\hline \multicolumn{8}{|c|}{ EYFP and tdTomato DG percentage area comparison } \\
\hline & TAM administration & $n$ & Test & Comparison & Statistic & $P$ & Significant \\
\hline $2 E$ & 3D Short & 3 & Two-way ANOVA & TAM $\times$ reporter & $F_{(2,6)}=8.880$ & 0.0161 & Yes* \\
\hline $2 E$ & 3D Long & 3 & & TAM & $F_{(2,6)}=11.32$ & 0.0092 & Yes** \\
\hline \multirow[t]{7}{*}{$2 E$} & $5 \mathrm{D}$ & 3 & & Reporter & $F_{(1,6)}=14.01$ & 0.0096 & Yes** \\
\hline & & & & Subject & $F_{(6,6)}=12.52$ & 0.0036 & Yes** \\
\hline & & & & EYFP & & tdTomato & \\
\hline & Post hoc & & Comparisons & Adjusted $P$ & Significant & Adjusted $P$ & Significant \\
\hline & Tukey's multiple & & 3D short vs 3D long & $\geq 0.9999$ & No & 0.9976 & No \\
\hline & comparisons & & $3 D$ short vs $5 D$ & 0.0009 & Yes*** & 0.0274 & Yes* \\
\hline & & & $3 D$ long vs 5D & 0.0009 & Yes**** & 0.0308 & Yes* \\
\hline
\end{tabular}

EYFP and tdTomato DG percentage area correlation

\begin{tabular}{|c|c|c|c|c|c|c|}
\hline Figure & TAM administration & $n$ & Test & Statistic & $P$ & Significan \\
\hline $2 F$ & All & 9 & $\begin{array}{l}\text { Pearson's } \\
\text { Correlation }\end{array}$ & $r_{(8)}=0.9157$ & 0.0005 & Yes*** \\
\hline \multicolumn{7}{|c|}{ EYFP + percentage area colocalization with tdTomato + area } \\
\hline & TAM administration & $n$ & Test & Statistic & $P$ & Significant \\
\hline $2 H$ & 3D Short & 3 & One-sample $t$ test & $t_{(2)}=35.87$ & 0.0008 & Yes*** \\
\hline $2 H$ & 3D Long & 3 & w/ comparison to & $t_{(2)}=13.15$ & 0.0057 & Yes** \\
\hline $2 H$ & $5 \mathrm{D}$ & 3 & $100 \%$ & $t_{(2)}=15.36$ & 0.0042 & Yes** \\
\hline
\end{tabular}

tdTomato + percentage area colocalization with EYFP + area

\begin{tabular}{|c|c|c|c|c|c|c|}
\hline Figure & TAM administration & $n$ & Test & Statistic & $P$ & Significant \\
\hline 21 & 3D Short & 3 & One-sample $t$ test & $t_{(2)}=119.7$ & 0.0001 & Yes***** \\
\hline 21 & 3D Long & 3 & w/ comparison to & $t_{(2)}=10.40$ & 0.0091 & Yes** \\
\hline 21 & $5 \mathrm{D}$ & 3 & $100 \%$ & $t_{(2)}=11.27$ & 0.0078 & Yes** \\
\hline
\end{tabular}

DG percentage of NSPCs with True + Signal

\begin{tabular}{|c|c|c|c|}
\hline Figure & TAM administration & $n$ & Test \\
\hline $4 B$ & 3D Short & 3 & Two-way ANOVA \\
\hline $4 B$ & 3D Long & 3 & \\
\hline $4 B$ & $5 D$ & 3 & \\
\hline
\end{tabular}

Post hoc

Tukey's multiple comparisons

DG percentage of NSPCs with True-Signal

\begin{tabular}{|c|c|c|c|}
\hline Figure & TAM administration & $n$ & Test \\
\hline $4 B$ & 3D Short & 3 & Two-way ANOVA \\
\hline $4 B$ & 3D Long & 3 & \\
\hline $4 B$ & 5D & 3 & \\
\hline
\end{tabular}

Post hoc

Tukey's multiple comparisons

Comparison
True signal $\times$ Tam
True signal
TAM
Subject

Statistic

$F_{(2,6)}=6.084$

$F_{(1,6)}=3.215$

$F_{(2,6)}=3.201$

$F_{(6,6)}=0.02808$

\section{Comparisons}

3D short vs 3D long

$3 D$ short vs $5 D$

$3 \mathrm{D}$ long vs $5 \mathrm{D}$

Comparison

True-Signal $\times$ TAM

True-Signal

TAM

Subject

Comparisons

3D short vs 3D long

$3 D$ short vs $5 D$

$3 D$ long vs $5 D$
Adjusted $P$

0.8875

0.0225

0.0516

Statistic

$F_{(2,6)}=6.084$

$F_{(1,6)}=3.215$

$F_{(2,6)}=3.201$

$F_{(6,6)}=0.02808$

Adjusted $P$

0.9389

0.0328

0.0179
$P$

0.0360

0.1231

0.1132

0.9998

Significant

No

Yes*

No

$P$

0.0360

0.1231

0.1132

0.9998

Significant

No

Yes*

Yes*

DG percentage of NSPCs with True \pm Signal

\begin{tabular}{|c|c|c|c|c|c|c|}
\hline Figure & TAM administration & $n$ & Test & Statistic & $P$ & Significant \\
\hline $4 C$ & 3D Short & 3 & One-way ANOVA & $F_{(2,6)}=3.201$ & 0.1132 & No \\
\hline $4 C$ & 3D Long & 3 & & $F_{(2,6)}=3.201$ & 0.1132 & No \\
\hline $4 C$ & $5 \mathrm{D}$ & 3 & & $F_{(2,6)}=3.201$ & 0.1132 & No \\
\hline
\end{tabular}

$* p<0.05, * * p<0.01, * * * p<0.001, * * * * p<0.0001$

assumed an experimentally-feasible $P\left(R_{r}\right)=0.5$ (i.e., $50 \%$ of target cells showing recombination-dependent reporter expression). In the ideal outcome for this experiment, where total true signal is $100 \%, 50 / 100$ cells would show simultaneous reporter expression and target gene recombination (Fig. 1B; Extended Data Fig. 1-1B, "theoretical ideal"). However, while reporter gene recombination is frequently used as an estimate of target gene 


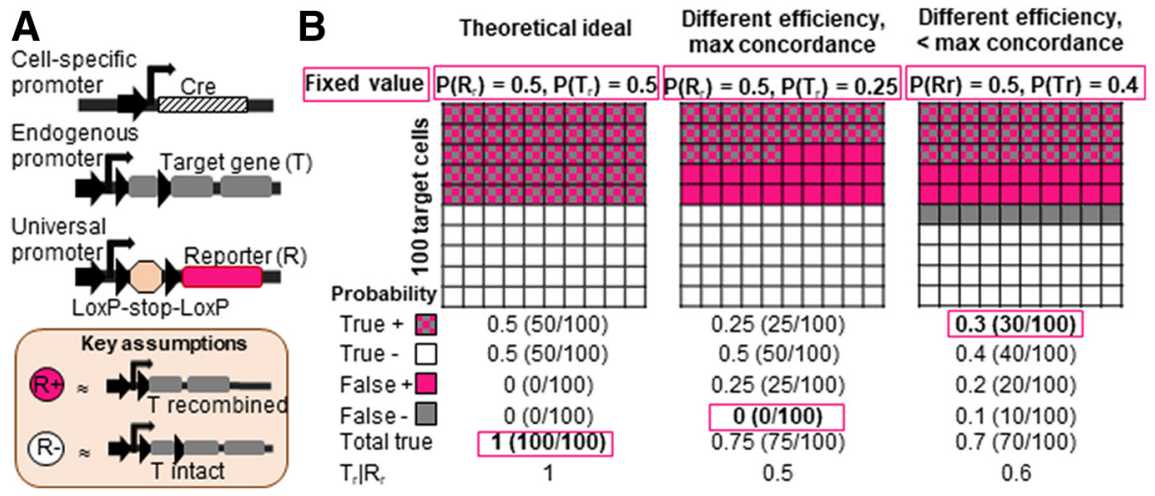

Figure 1. Theoretical probabilistic models reveal potential for error in a common paradigm for testing cell autonomous gene function. $\boldsymbol{A}$, Cell autonomous function of genes is frequently investigated using a cell-specific Cre-loxP system where expression of reporter protein from a stop-floxed reporter construct is assumed to reliably indicate target gene recombination within a single cell. Here, a generic schematic of the genetics used and assumptions employed in this common paradigm are shown. $\boldsymbol{B}$, The probabilities of true and false reporter signals are shown for three hypothetical scenarios with 100 target cells for visualization. All three scenarios assume an experiment where gene recombination occurs only in the presence of Cre, reporter protein expression is observed in $50 \%$ of target cells $\left[P\left(R_{r}\right)=0.5\right]$, and reporter recombination is greater than or equal to target gene recombination $\left[P\left(R_{r}\right) \geq\right.$ $P\left(T_{r}\right)$ ]. The numbers in red boxes represent given values that are then used to determine the remaining probabilities. The "theoretical ideal" represents an ideal scenario where reporter gene recombination and target gene recombination overlap perfectly. The "different efficiency, max concordance" scenario shows a case where target gene recombines with less efficiency than the reporter, but shows the maximum possible concordance with reporter recombination given that constraint. The "different efficiency, < max concordance" scenario shows a case where target gene recombination is mildly less efficient than reporter gene recombination and concordance is moderately suboptimal, with $75 \%$ of target recombined cells also showing reporter recombination. In the hypothetical deviations from ideal, the probability of reporter expression accurately predicting target gene recombination $\left[P\left(T_{r} \mid R_{r}\right)\right]$ is 0.5 and 0.6 , respectively. These examples demonstrate that even mild deviation from ideal can introduce possible substantial error in using reporter gene recombination as an indicator of target gene recombination in the same cell. See also Extended Data Figure 1-1.

Figure Contributions: Elizabeth Diana Kirby developed theoretical models and made figures.

recombination, there is ample evidence that efficiency of Cre-loxP recombination varies from gene to gene (Long and Rossi, 2009; Sun et al., 2014; Gray et al., 2017). We therefore examined the effect of setting $P\left(R_{r}\right)=0.5$ but $P$ $\left(T_{r}\right)=0.25$. Using the best possible scenario where target gene recombination occurs only in reporter gene recombined cells [i.e., $P($ false -$)=0$ ], we found that $P\left(T_{r} \mid R_{r}\right)=0.5$, meaning that identifying a cell as reporter positive only yields a $50 \%$ chance that the cell is also target gene recombined (Fig. 1B; Extended Data Fig. 1-1C, "different efficiency, max concordance").

We next estimated the effect of a less dramatic difference between $P\left(R_{r}\right)$ and $P\left(T_{r}\right)$ but when mixed with moderately suboptimal recombination concordance between the two genes within one cell. Using $P\left(T_{r}\right)=0.4$ and assuming that $75 \%$ of target recombined cells are also reporter recombined [i.e., $P\left(T_{r}\right) * 0.75=P($ true +$)$, we found that observing a reporterexpressing cell only yielded a $60 \%$ chance that that cell was also target gene recombined [i.e., $\left.P\left(T_{r} \mid R_{r}\right)=0.6\right]$. Observing a reporter-negative cell in this case yielded a $10 \%$ chance that that cell was target gene recombined $[\mathrm{P}($ false -$)=0.1$; Fig. $1 B$; Extended Data Fig. 1-1D, "different efficiency, $<\max$ concordance"]. These findings suggest that seemingly mild variation in recombination efficiency and suboptimal concordance rates for gene recombination could introduce substantial error in the method of using reporter expression as a marker of target gene recombination within a single cell.

To test the accuracy of using Cre-induced recombination of one gene to predict recombination of another gene in an in vivo experimental paradigm, we combined
NestinCreER ${ }^{\top 2}$ mice with two commonly-used conditional reporter lines: Rosa-stop-floxed-EYFP (Srinivas et al., 2001) and Rosa-CAG-stop-floxed-tdTomato (Madisen et al., 2010; Fig. 2A). NestinCreER ${ }^{\mathrm{T} 2}$ mice express a TAM-sensitive Cre recombinase that drives recombination of floxed sequences in Nestin-expressing NSPCs in the adult brain (Lagace et al., 2007). The floxed sequences used, Rosastop-floxed-EYFP and Rosa-CAG-stop-floxed-tdTomato genes, are both inserted in the Rosa locus, although the tdTomato construct also includes an additional CAG promoter that the EYFP construct does not (Fig. 2A; Srinivas et al., 2001; Madisen et al., 2010).

First, to verify the TAM-dependency of reporter gene recombination in this model, adult NestinCreER ${ }^{\top 2}$; Rosa (EYFP/tdTomato) mice were injected with oil (vehicle) or TAM once per day for $5 \mathrm{~d}$. This TAM dosing schedule is widely-used and leads to efficient and specific recombination of floxed genetic sequences in NSPCs in adult NestinCreER ${ }^{\text {T2 }}$ mice (Sun et al., 2014). As expected, oilinjected mice exhibited negligible ectopic recombination of either reporter gene (Extended Data Fig. 2-1A) while TAM administration induced robust fluorescent reporter expression in DG NSPCs (Extended Data Fig. 2-1B). These findings indicate that recombination of both reporter genes is tightly dependent on TAM, as found in previous studies (Imayoshi et al., 2006; Kim et al., 2011; Yang et al., 2015).

We next examined the population-level efficiencies of recombination in both fluorescent reporter genes to determine if recombination frequency in one gene is a 


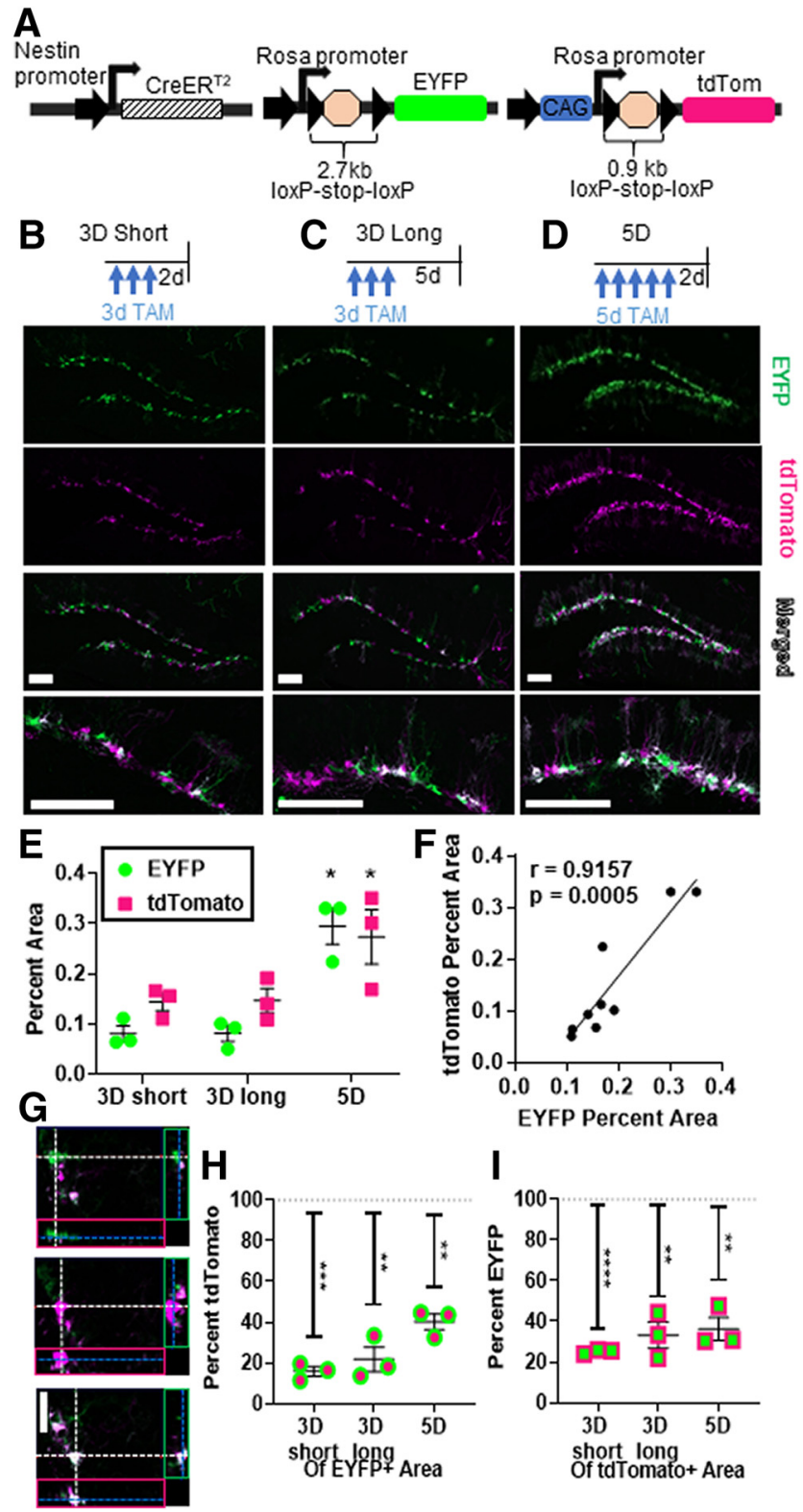

Figure 2. Recombination-dependent fluorescent reporter gene expression is correlated over the whole SGZ, but frequently fails to co-localize. $\boldsymbol{A}, \mathrm{A}$ schematic of the transgenic mouse model employed in our experiments where we combined NestinCreER ${ }^{\text {T2 }}$ mice Rosa-stop-floxed-EYFP and Rosa-CAGstop-floxed-tdTomato mice. Mice were then submitted to three TAM administration conditions, 3D short $(\boldsymbol{B})$, 3D long $(\boldsymbol{C})$, and 5D ( $\boldsymbol{D}$; top, representative timeline of TAM injections and recovery; bottom, immunostaining of EYFP and tdTomato in SGZ; scale bars: $100 \mu \mathrm{m})$. E, EYFP+ and tdTomato+ DG percent area was compared by two-way ANOVA in 3D short, 3D long, and 5D mice. Tukey's post hoc comparison within reporter type; $* p<0.05, * * p<0.01, * * * p<0.001$ versus $5 D$. $\boldsymbol{F}$, EYFP+ and tdTomato + DG percent area were correlated in all mice. $R$, p Pearson's correlation. G, Representative orthogonal images: immunostaining and imaging of EYFP+, tdTomato + , and EYFP +tdTomato+ cells in the SGZ. Scale bar: $20 \mu \mathrm{m}$. $\boldsymbol{H}$, EYFP + colocalization in tdTomato + area $(\boldsymbol{H})$ and tdTomato + colocalization in EYFP + area $(\boldsymbol{I})$ were compared with theoretical $100 \%$ colocalization; $n=3$ mice per group. Data are shown as general predictor of recombination in a separate gene. To create a range of recombination rates, NestinCreER ${ }^{\mathrm{T} 2}$; Rosa(EYFP/tdTom) mice were submitted to one of three different TAM administration and recovery protocols: $3 \mathrm{~d}$ of TAM with $3 d$ of recovery (3D short), $3 d$ of TAM with $5 d$ of recovery (3D long) and $5 \mathrm{~d}$ of TAM with $3 \mathrm{~d}$ of recovery (5D), the standard TAM regimen (Fig. 2B-D). Analysis of the percent area occupied by fluorescent reporter proteins in the DG confirmed that a range of recombination rates was achieved, with 5D TAM leading to the highest level of reporter expression $\left(F_{(2,6)}=11.32, p=0.0092\right.$; two-way ANOVA; $p<0.05$ Tukey's; Fig. $2 E$ ). Across all three TAM groups, there was a strong and significant correlation between tdTomato and EYFP expression in the DG of TAM injected mice $\left(r_{(8)}=0.9157, p=0.0005\right.$; Pearson's correlation; Fig. 2F). These findings suggest that mice with high recombination of one reporter gene also have high recombination of the other and support the possibility that recombination of one gene might be predictive of recombination in a second gene.

To examine reporter coexpression within single cells, we quantified EYFP and tdTomato antibody-amplified fluorescence co-localization in 1- $\mu \mathrm{m}$ z-stack image series from adult DG. In these images, cells expressing EYFP alone appear green and cells expressing tdTomato alone appear magenta, while cells expressing both fluorescent reporters appear white (Fig. 2G). Qualitative assessment of reporter expression revealed representation of each of these three possible colocalization phenotypes (Fig. 2G). Using an automated co-localization tool to quantify reporter overlap, we found that EYFP+ percent area colocalization with tdTomato + signal ranged from $15.84 \%$ in 3D short mice and $21.77 \%$ in $3 \mathrm{D}$ long mice to $36.71 \%$ in 5D mice, all of which were significantly less than $100 \%$ (3Ds: $t_{(2)}=35.87, p=0.00083 \mathrm{DI}: t_{(2)}=13.15, p=0.0057$ 5D: $t_{(2)}=15.36, p=0.0042$; one-sample $t$ test comparison to $100 \%$; Fig. $2 H$ ). The converse, tdTomato+ percent area of EYFP + area, showed slightly higher colocalization frequencies: 3D short $25.14 \%$, 3D long $33.22 \%$, and 5D $36.30 \%$, although all still significantly less than $100 \%$ (3Ds: $t_{(2)}=119.7, p=0.00013 \mathrm{DI}: t_{(2)}=10.40, p=0.0091$ 5D: $t_{(2)}=11.27, p=0.0078$; one-sample $t$ test comparison to $100 \%$; Fig. 2/). These results suggest that the fluorescent markers may not be coexpressed in the same NSPCs with high frequency.

To quantify recombination frequencies within NSPC subpopulations in the SGZ, we used immunofluorescent labeling for phenotypic markers to identify reporter expression in RGLs and IPCs (Fig. 3A,B). RGLs were identified based on a cell body in the SGZ with a GFAP+ radial process extending through the granule cell layer (Lugert et al., 2010; Fig. 3A). IPCs were identified as $\mathrm{Ki}-67+$ nuclei in the SGZ (Mandyam et al., 2007; Fig. 3B). Recombination frequency

\section{continued}

mean \pm SEM; $* p<0.05, * * p<0.01, * * * p<0.001, * * * * p<$ 0.0001 .

Figure Contributions: Tyler Joseph Dause ran experiments and analyzed data. Tyler Joseph Dause and Elizabeth Diana Kirby made figure. 


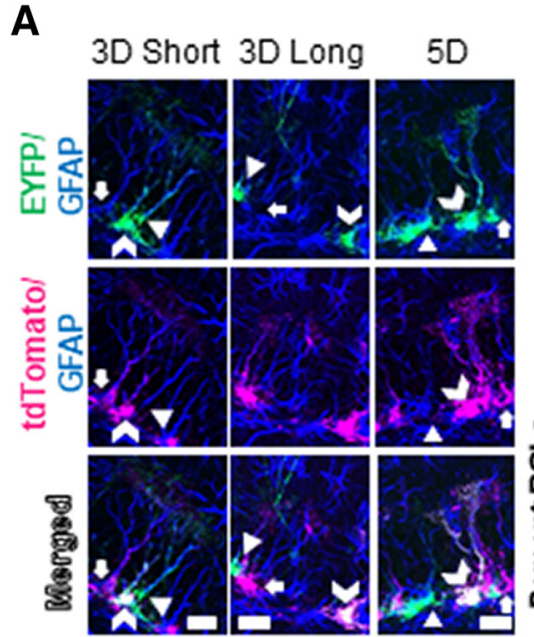

B

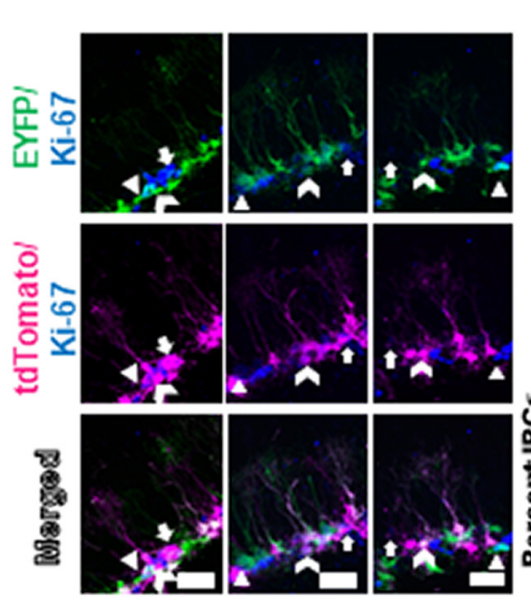

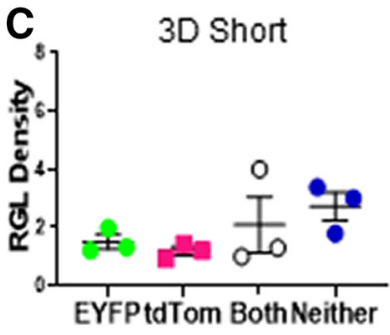

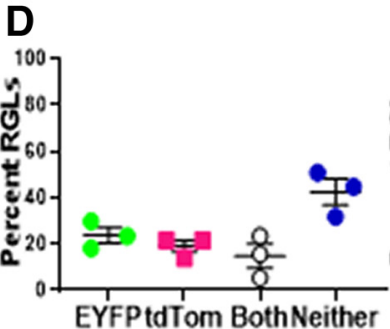

E
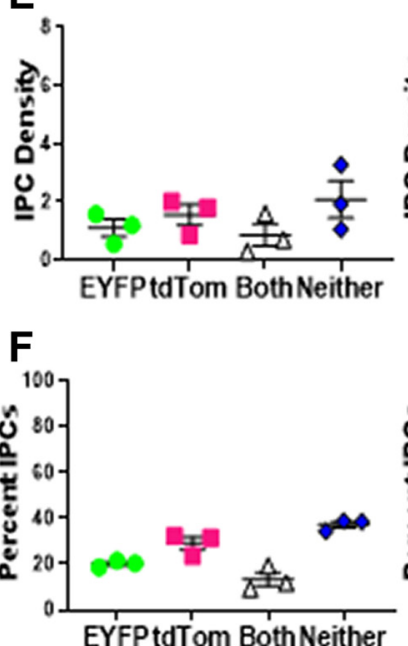
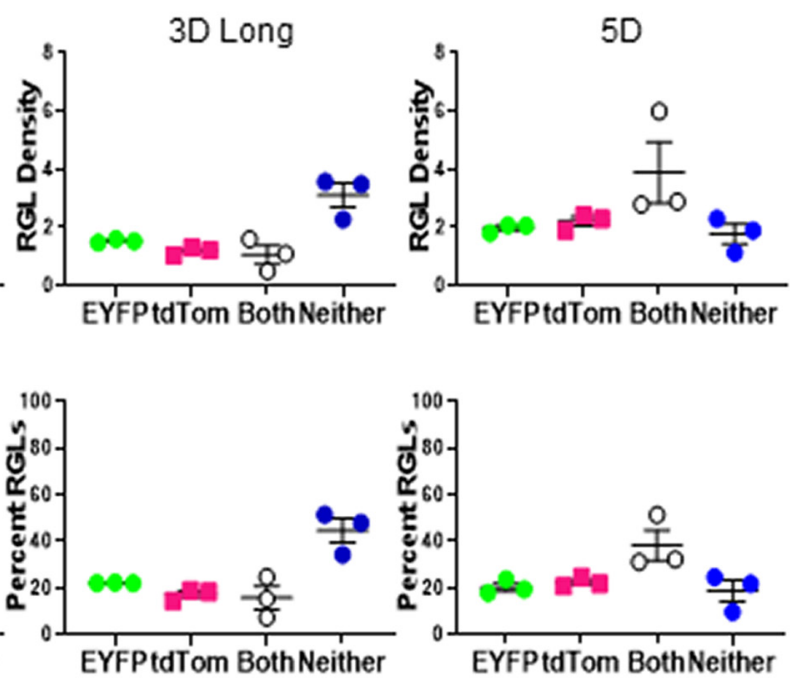

Figure 3. Recombination-dependent fluorescent reporter gene expression within RGLs and IPCs frequently fails to co-localize in the same cells. $\boldsymbol{A}$, SGZ RGLs were identified by immunostaining of EYFP, tdTomato, and GFAP. Scale bars: $20 \mu \mathrm{m}$. Arrow head = EYFP +/tdTomato- RGL. Arrow = EYFP-/tdTomato + RGL. Chevron = EYFP+/tdTomato + RGL. $\boldsymbol{B}$, SGZ IPCs were identified by immunostaining of EYFP, tdTomato and Ki-67. Scale bars: $20 \mu \mathrm{m}$. Arrow head = EYFP+/tdTomato- IPC. Arrow $=$ EYFP-/tdTomato + IPC. Chevron = EYFP+/tdTomato + IPC. Density $(\boldsymbol{C})$ and percentage $(\boldsymbol{D})$ of SGZ GFAP+ RGLs coexpressing EYFP only(EYFP), tdTomato only(tdTom), EYFP + and tdTomato + (both), or neither are shown. Density $(\boldsymbol{E})$ and percentage $(\boldsymbol{F})$ of SGZ Ki67+ IPCs coexpressing EYFP, tdTom, both, or neither are shown; $n=3$ mice per group. Data are shown as mean \pm SEM. Density represented as cells per area, scale $\times 10^{-4}$. See also Extended Data Figure 3-1.

Figure Contributions: Tyler Joseph Dause ran experiments and analyzed data. Tyler Joseph Dause and Elizabeth Diana Kirby made figure.

of each reporter gene individually was similar to that reported in previous studies using NestinCreER ${ }^{\mathrm{T} 2}$ mice (Extended Data Fig. 3-1A-D). In addition, similar to our findings using fluorescent reporter area, recombination rates of EYFP and tdTomato reporter genes were positively associated $\left[r_{(8)}=0.9381, p=0.0002\right.$; Pearson's correlation (Extended Data Fig. 3-1B); $r_{(8)}=0.6308, p=0.0685$; Pearson's correlation (Extended Data Fig. 3-1D)]. However, at the single-cell level, we observed substantial populations of EYFP+/tdTomato- and EYFP-/tdTomato+ RGLs and IPCs in all groups (Fig. 3C-F), suggesting prevalent mismatch in recombination of the two genes in individual NSPCs.
To examine the consequences of the rates of single reporter recombination observed in our in vivo experiments, we applied the reporter expression frequencies observed in the 5D TAM group to a theoretical experiment where tdTomato $\left(T_{r}\right.$, equivalent of $R_{r}$ in Fig. 1$)$ is used to predict recombination in the EYFP reporter gene $\left(E_{r}\right.$, equivalent of $T_{r}$ in Fig. 1). In the 5D TAM group, the conditional probability $P\left(E_{r} \mid T d_{r}\right)$ was 0.63 or 0.58 for RGLs and IPCs, respectively, meaning that there was a $63 \%$ or $58 \%$ probability that an RGL or IPC identified as tdTomato+ also showed EYFP expression (Fig. 4A). The converse, $\mathrm{P}\left(\mathrm{E}_{\mathrm{r}}{ }^{\prime} \mid \mathrm{Td}_{\mathrm{r}}{ }^{\prime}\right)$, was 0.49 and 0.50 for RGLs and IPCs, respectively, meaning that identifying an RGL or IPC as tdTomato negative only 
A

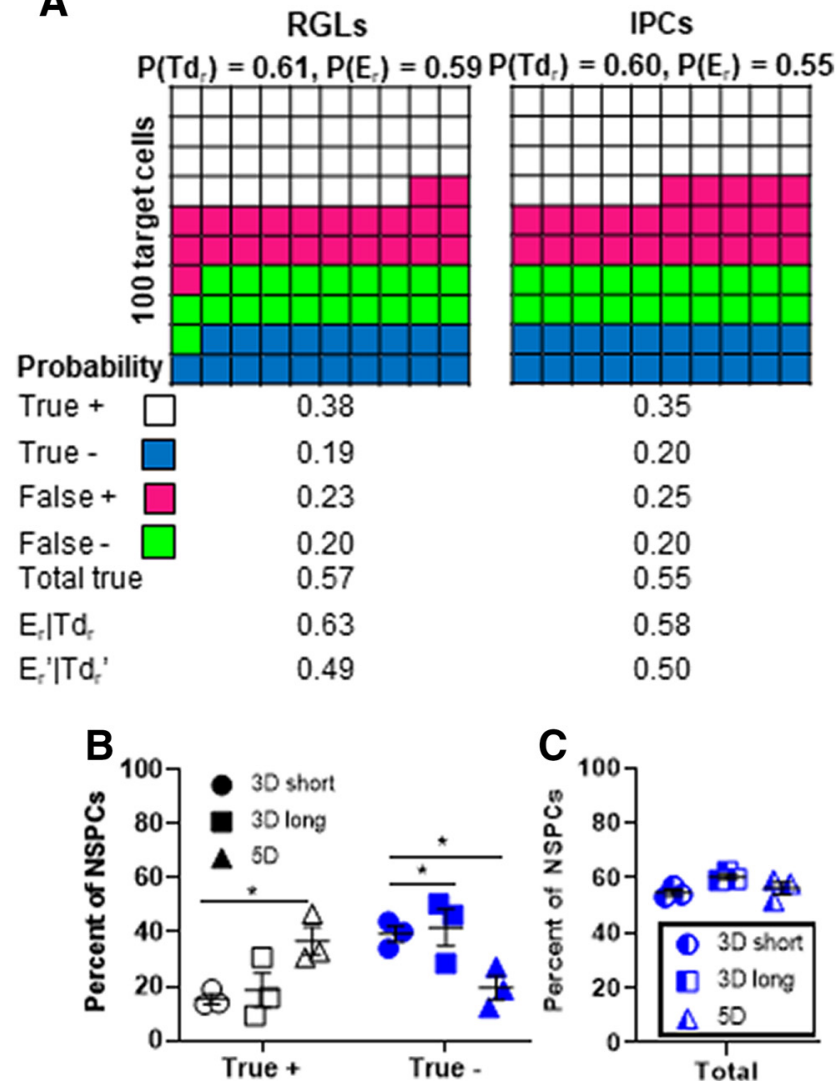

Figure 4. Using expression of one reporter to predict expression of the other results in high false signals similarly across TAM protocols. $\boldsymbol{A}$, The mean observed recombination frequencies from the 5D TAM group are represented here as reporter expression in 100 hypothetical target cells, either RGLs or IPCs. The probabilities of true and false signals are given as if tdTomato expression is being used to predict EYFP expression. $\boldsymbol{B}$, The percent of RGLs and IPCs combined (total NSPCs) that show recombination in both reporter genes (true + ) or neither (true -) is shown for three TAM groups. $\boldsymbol{C}$, The total true signal between the three TAM groups is shown; $n=3$ mice per group. Data shown are mean \pm SEM; $* p<0.05$ determined by twoway ANOVA. See also Extended Data Figures 4-1, 4-2.

Figure Contributions: Elizabeth Diana Kirby performed statistical analyses and made figures.

yielded a $49 \%$ or $50 \%$ probability that the cell was also EYFP negative (Fig. 4A). Comparing true positive and true negative signal in NSPCs across all three TAM protocols revealed that TAM protocol significantly interacted with the type of true signal (positive vs negative; $F_{(2,6)}=6.084$, $p=0.0360$; two-way ANOVA; Fig. 4B; Extended Data Fig. $4-1 A)$. Post hoc Tukey's comparisons revealed that $5 \mathrm{D}$ TAM mice showed higher true positive signal than 3D short mice $(p=0.0225)$, but also lower true negative signal than 3D short or 3D long groups $(p=0.0328$, $p=0.0179$; Fig. $4 B$ ). When total true signal was summed, no difference was observed between TAM protocols $\left(F_{(2,6)}=3.201, p=0.1132\right.$; one-way ANOVA Fig. $\left.4 C\right)$. Findings were similar when RGLs and IPCs were considered separately (Extended Data Fig. 4-1B-E). These findings suggest that despite a wide range of recombination frequencies, none of the tested TAM protocols led to better recombination concordance within single cells than the others.

Although we focus on NSPCs in the SGZ, NSPCs are also found in the SVZ. Similar to the SGZ, in the SVZ, TAM administration induced robust expression of both reporters that was correlated at the population level $\left(r_{(9)}=\right.$ $0.7908, p=0.0077$; Pearson's correlation; Extended Data Fig. 4-2A,B). However, as with the DG, we found significant divergence of expression at the single-cell level. TdTomato + colocalization within the EYFP+ area (57\%) was significantly less than $100 \%$ in the SVZ $\left(t_{(2)}=15.08\right.$, $p<0.0001$, one-sample $t$ test comparison to $100 \%)$ as was EYFP + percent area of tdTomato + area $\left(40 \%\right.$; $t_{(2)}=$ 9.115, $p=0.0001$, one-sample $t$ test comparison to 100\%; Extended Data Fig. 4-2C,D). These findings support and extend those from the SGZ by again showing that recombination of one fluorescent reporter gene may not accurately predict recombination of another at the single-cell level in adult NSPCs.

\section{Discussion}

Cell-specific gene manipulation is a powerful tool for investigating complex cellular mechanisms like neurogenesis in vivo. Here, we examine a common experimental model to assess cell autonomous function in which Cre-induced recombination of a stop-floxed fluorescent reporter is used to predict recombination of a target gene at the single-cell level. The reliability of this model is contingent on the assumption that transiently-activated Cre induces independent recombination events equivalently within a single cell. We first examined this experimental paradigm with probabilistic calculations, which revealed that mild variation in recombination efficiencies and discordance between recombination in a fluorescent reporter gene and a target gene could lead to substantial error in identifying cells with target gene recombination. These data led us to examine the accuracy of this methodology in a mouse model. In our model, we quantified TAM-induced, Cre-dependent recombination of two stop-floxed fluorescent reporter constructs in adult NSPCs. Our findings revealed that, while both reporters were highly expressed in NSPCs and global recombination rates of the two reporters were correlated, expression of either fluorescent reporter was an inaccurate predictor of expression of the other in individual NSPCs. Although different Cre drivers and different reporters may yield different results from those obtained here, our data suggest that it may not be reliable to assume that recombination of one gene is predictive of recombination of a second gene within single cells using TAM-sensitive Cre recombinases to drive recombination.

We began our investigation by modeling the theoretical accuracy of using Cre-induced recombination of one gene to predict recombination in another gene. In our model, stop-floxed fluorescent reporter gene (Gene R) expression is used as a marker of target gene (Gene T) recombination for analyses of cell autonomous target gene function. Using reasonable experimental expectations in a generic experimental design, we found that presence of a reporter protein $\mathrm{R}$ could be a poor predictor of target 
gene recombination. Even in a case where maximal concordance of recombination occurs, if a 2-fold difference in overall recombination rates were present, reporter presence would accurately predict target gene recombination in only $50 \%$ of cells. A previous study using three separate stop-floxed reporters each combined with the same NestinCreER ${ }^{\text {T2 }}$ drivers revealed recombination efficiencies ranging over 10-fold (Sun et al., 2014), suggesting that our allowance of a 2-fold difference in efficiency is well within the experimentally probable range. Our theoretical predictions therefore suggest that using stopfloxed fluorescent reporter proteins to identify target gene recombined cells could yield false signal at a high frequency even if the only source of error were difference in overall recombination efficiency.

Next, we examined the results of our theoretical models in an in vivo experimental paradigm where we used two separate fluorescent reporters as indicators of two possible recombination events within a single cell. We used a highly-cited, NSPC-specific and efficient NestinCreER ${ }^{\text {T2 }}$ line to drive recombination of two highly-cited stop-floxed fluorescent reporters. In these mice, we found that presence of one reporter accurately predicted presence of the other reporter in only $50-60 \%$ of NSPCs. While different reporters and different Cre-drivers may reveal different results, our data suggest that the assumption that two, independent recombination events show high concordance within a cell may be false, even in mouse lines used in hundreds of studies.

The likelihood of Cre-mediated recombination events can be influenced by several factors: the paradigm of Cre induction, features of the Cre recombinase, and features of the loxP-flanked genes. First, Cre induction and resulting loxP recombination can depend on the dose, frequency and route of TAM administration, as well as the delay between TAM and tissue harvest. For example, here we show that five TAM injections significantly increased global fluorescent reporter recombination rates compared with three TAM injections, as one would expect. However, of three different TAM injection protocols that varied in number of injections and delay before harvest, we found that no one of the protocols led to higher concordance in recombination (or lack of recombination) in the two reporter genes than the others. Nonetheless, other dosing schedules or routes (such as orally) may yield different results.

Features of the Cre recombinase can also impact likelihood of loxP recombination. Previous work shows that efficiency of different $\mathrm{CreER}^{\mathrm{T} 2}$ lines at driving recombination events can vary substantially (Sun et al., 2014). These differences may be due to the sequence of the recombinase gene itself or due to its insertion location in the genome. Use of inducible Cre, as opposed to a constitutively active form of Cre, also likely decreases recombination efficiency. The NestinCreER ${ }^{\text {T2 }}$ line used in the present study is one of the lower efficiency, inducible NSPC-targeted Cre lines of those available (Sun et al., 2014). A NestinCreER ${ }^{\text {T2 }}$ line with higher efficiency would likely show greater concordance in recombination events within single cells. However, if the goal of a study is to compare recombined and non- recombined cells, driving recombination to near $100 \%$, while it would yield better concordance rates across genes, would eliminate the non-recombined cell population of comparison. The importance of recombination specificity also must be considered when selecting a Cre-driver line. For example, although the NestinCreER ${ }^{\mathrm{T} 2}$ line used here has low efficiency, it is also more specific to NSPCs than the comparable lines with higher efficiency (Sun et al., 2014).

The features of loxP flanked constructs can also impact the likelihood of Cre-lox recombination. In the present study, paired fluorescent reporter genes were located in identical Rosa loci, making them more similar than most reporter-target combinations. However, the tdTomato construct contained a CAG promotor that the EYFP construct lacked. The distance between loxP sites also differed, with the tdTomato construct having $0.9 \mathrm{~kb}$ between loxP sites while the EYFP construct contained $2.7 \mathrm{~kb}$ between loxP sites. The above factors could lead to preferential recombination of tdTomato expression over EYFP, which we observed signs of in the quantification of fluorescent area. However, manual cell counts did not show greater detection of one reporter over the other, and both EYFP+/tdTomato- and EYFP-/tdTomato+ single cells were found, suggesting that our findings of poor concordance are not driven solely by lower recombination rates in one reporter gene compared with the other. In most experimental paradigms designed to test cell autonomous gene function, expression of a stop-floxed fluorescent reporter is assumed to imply recombination of a separate target gene, which has a different promoter, different loxP site separation and different gene sequence than the reporter construct. Therefore, while the two reporters used here are not identical, they share many similarities that should drive single-cell concordance rates to be higher than in a typical experiment where target gene and reporter are less related. Our findings therefore suggest that even with similar overall recombination rates and similar genetic locations, there can be substantial divergence in recombination events within a single cell.

A separate issue from gene recombination is detection of recombination. Fluorescent reporter expression, although present, may not reach the threshold for detection in every cell. To combat this, we performed both manual and automated data analyses, which both yielded similar findings. However, using more sensitive microscopy methods for detecting fluorescent markers may reveal a higher percentage of overlap in reporter expression. The relative intensity of EYFP signal versus tdTomato signal may also differ, driving different detection rates of each reporter. Antibodies against EYFP and tdTomato were used in the present work to increase detection of both reporters and only a slight increase in tdTomato detection was found over EYFP in data derived from fluorescent area. The concern of fluorescence detection would also apply to empirical studies of gene recombination function and may be a source of error there as well.

A relevant question raised by our theoretical and experimental results is how much error is acceptable in studies of cell autonomous gene function. Using reporter protein 
presence (or absence) to define cells as target gene recombined (or intact) is effectively dividing cells in to separate experimental groups. We therefore suggest that the error acceptable for reporter and target gene recombination concordance should be similar to error researchers would deem acceptable in identifying animals in different experimental groups. When considering the standard for error used in biological statistics, we suggest that a reasonable experimental expectation of this model would be gene recombination concordance of at least $95 \%$. The observed $50-60 \%$ accuracy of one reporter predicting recombination of another reporter found in the present study falls far short of this threshold, and also is likely much lower than what most investigators would consider acceptable error in identifying treatment groups in a randomized study.

Given the large number of CreER lines, fluorescent reporters, and individual floxed genes available, it may be impossible to determine how widely the present findings apply. Different Cre driver-reporter gene-target gene combinations could substantially differ from the present results, yielding more or less reliable concordance in recombination between separate genes. Ideally, the concordance of target gene and reporter gene recombination would be tested in each specific transgenic model where it is being applied for study of cell autonomous gene recombination. This process would require some form of alternative verification of target gene recombination such as immunohistochemistry or in situ hybridization. However, stop-floxed reporters are typically used precisely because other methods of identifying target gene-recombined cells are lacking. We therefore suggest that these results present a cautionary note and that given the potential for error in this method, alternative methods of studying cell autonomous gene function should be considered.

There are several alternative methods for identifying the effects of cell autonomous gene manipulation. One paradigm that still uses inducible Cre is to integrate fluorescent reporters in the target genomic sequence. Such models will likely require creation of new transgenic mice for many target genes, as few existing models include linked reporters in this fashion. An additional alternative method is to deliver transgenes plus cleavably-linked fluorescent reporters using viral vectors that target expression to specific cell populations based on viral serotype or cell-specific promoters. For example, certain adeno-associated viruses show preference for infecting specific NSPC subclasses and can be used to manipulate gene function in adulthood after stereotaxic delivery (Crowther et al., 2018).

In addition to tracking recombination in single cells, stop-floxed reporters are also frequently used to identify the cell population targeted by a Cre-driver line (i.e., population specificity), as well as overall recombination efficiencies. We found that both tdTomato and EYFP expression were similarly concentrated in adult NSPCs, suggesting that cell population specificity of recombination was similar regardless of reporter. We also found a strong correlation between recombination efficiency of the two reporters in individual mice, suggesting that, at least in this case, reporters could reliably predict high-recombined subjects versus low-recombined subjects. However, it is unlikely that absolute recombination efficiencies can be extrapolated across genes due to the well-documented differences in loxP recombination probability across genomic loci (Long and Rossi, 2009; Gray et al., 2017).

Our findings do not necessarily undermine previous studies which have identified cell autonomous gene functions using stop-floxed reporters as a marker of target gene recombined cells (Zhou et al., 2018; Zimmermann et al., 2018; Franz et al., 2019; Zhang et al., 2019). Aside from the possibility that other reporter-target combinations show better recombination concordance than the two genes used in this study, if target gene recombination has a large cell-autonomous effect, it may still be detectable in models with suboptimal reporter-target recombination concordance due to the subpopulation of cells in which fluorescent protein presence is a true signal. False negative and false positive signals like those observed in this model, if present in other models, would be most likely to obscure smaller cell autonomous effects and lead to false negative findings when cell autonomous gene functions are in fact present.

In summary, our findings suggest that models of inducible gene manipulation combined with a ubiquitously-expressed stop-floxed fluorescent reporter could be unreliable in their ability to identify cell autonomous effects at a single-cell level in vivo. We support this finding with theoretical probability estimates of a generic Cre-lox experiment and experimental data from one example Cre-lox system. Future work is necessary to create reliable and cost-effective models that can be easily applied to the study of cell-autonomous effects across many target genes. Such models will be imperative for studying molecular mediators of complex cellular processes such as adult neurogenesis.

\section{References}

Bolte S, Cordelières FP (2006) A guided tour into subcellular colocalization analysis in light microscopy. J Microsc 224:213-232.

Crowther AJ, Lim SA, Asrican B, Albright BH, Wooten J, Yeh CY, Bao H, Cerri DH, Hu J, lan Shih YY, Asokan A, Song J (2018) An adeno-associated virus-based toolkit for preferential targeting and manipulating quiescent neural stem cells in the adult hippocampus. Stem Cell10:1146-1159.

Feil S, Valtcheva N, Feil R (2009) Inducible Cre mice. Methods Mol Biol 530:343-363.

Franz $\mathrm{H}$, Villarreal $\mathrm{A}$, Heidrich $\mathrm{S}$, Videm $\mathrm{P}$, Kilpert $\mathrm{F}$, Mestres I, Calegari F, Backofen R, Manke T, Vogel T (2019) DOT1L promotes progenitor proliferation and primes neuronal layer identity in the developing cerebral cortex. Nucleic Acids Res 47:168-183.

Gage FH (2000) Mammalian neural stem cells. Science 287:14331438.

Gray LT, Yao Z, Nguyen TN, Kim TK, Zeng H, Tasic B (2017) Layerspecific chromatin accessibility landscapes reveal regulatory networks in adult mouse visual cortex. Elife 6:e21883.

Imayoshi I, Ohtsuka T, Metzger D, Chambon P, Kageyama R (2006) Temporal regulation of Cre recombinase activity in neural stem cells. genesis 44:233-238.

Kempermann G (2015) Adult neurogenesis: An evolutionary perspective. Cold Spring Harb Perspect Biol 8:a018986.

Kim EJ, Ables JL, Dickel LK, Eisch AJ, Johnson JE (2011) Ascl1 (Mash1) defines cells with long-term neurogenic potential in 
subgranular and subventricular zones in adult mouse brain. PLoS One 6:e18472.

Kirby ED, Kuwahara AA, Messer RL, Wyss-Coray T (2015) Adult hippocampal neural stem and progenitor cells regulate the neurogenic niche by secreting VEGF. Proc Natl Acad Sci USA 112:4128-4133.

Lagace DC, Whitman MC, Noonan MA, Ables JL, DeCarolis NA, Arguello AA, Donovan MH, Fischer SJ, Farnbauch LA, Beech RD, DiLeone RJ, Greer CA, Mandyam CD, Eisch AJ (2007) Dynamic contribution of Nestin-expressing stem cells to adult neurogenesis. J Neurosci 27:12623-12629.

Long MA, Rossi FMV (2009) Silencing inhibits Cre-mediated recombination of the Z/AP and Z/EG reporters in adult cells. PLoS One 4: e5435.

Lugert S, Basak O, Knuckles P, Haussler U, Fabel K, Götz M, Haas CA, Kempermann G, Taylor V, Giachino C (2010) Quiescent and active hippocampal neural stem cells with distinct morphologies respond selectively to physiological and pathological stimuli and aging. Cell Stem Cell 6:445-456.

Madisen L, Zwingman TA, Sunkin SM, Oh SW, Zariwala HA, Gu H, $\mathrm{Ng}$ LL, Palmiter RD, Hawrylycz MJ, Jones AR, Lein ES, Zeng $\mathrm{H}$ (2010) A robust and high-throughput Cre reporting and characterization system for the whole mouse brain. Nat Neurosci 13:133140.

Mandyam CD, Harburg GC, Eisch AJ (2007) Determination of key aspects of precursor cell proliferation, cell cycle length and kinetics in the adult mouse subgranular zone. Neuroscience 146:108-122.

Semerci F, Maletic-Savatic M (2016) Transgenic mouse models for studying adult neurogenesis. Front Biol (Beijing) 11:151-167.

Srinivas S, Watanabe T, Lin CS, William CM, Tanabe Y, Jessell TM, Costantini $F$ (2001) Cre reporter strains produced by targeted insertion of EYFP and ECFP into the ROSA26 locus. BMC Dev Biol $1: 4$.

Sun MY, Yetman MJ, Lee TC, Chen Y, Jankowsky JL (2014) Specificity and efficiency of reporter expression in adult neural progenitors vary substantially among nestin-CreER(T2) lines. J Comp Neurol 522:1191-1208.

Tannenholz L, Hen R, Kheirbek MA (2016) GluN2B-containg NMDA receptors on adult-born granule cells contribute to the antidepressant action of fluoxetine. Front Neurosci 10:242.

Yang SM, Alvarez DD, Schinder AF (2015) Reliable genetic labeling of adult-born dentate granule cells using Ascl1CreERT2 and GlastCreERT2 murine lines. J Neurosci 35:15379-15390.

Zhang H, Sathyamurthy A, Liu F, Li L, Zhang L, Dong Z, Cui W, Sun X, Zhao K, Wang H, Ho HYH, Xiong WC, Mei L (2019) Agrin-Lrp4Ror2 signaling regulates adult hippocampal neurogenesis in mice. Elife 8:e45303.

Zhao C, Deng W, Gage FH (2008) Mechanisms and functional implications of adult neurogenesis. Cell 132:645-660.

Zhou Y, Bond AM, Shade JE, Zhu Y, Davis C-H, Wang X, Su Y, Yoon K-J, Phan AT, Chen WJ, Oh JH, Marsh-Armstrong N, Atabai K, Ming G-L, Song H (2018) Autocrine Mfge8 signaling prevents developmental exhaustion of the adult neural stem cell pool. Cell Stem Cell 23:444-452.e4.

Zimmermann T, Maroso M, Beer A, Baddenhausen S, Ludewig S, Fan W, Vennin C, Loch S, Berninger B, Hofmann C, Korte M, Soltesz I, Lutz B, Leschik J (2018) Neural stem cell lineage-specific cannabinoid type-1 receptor regulates neurogenesis and plasticity in the adult mouse hippocampus. Cereb Cortex 28:4454-4471. 